OPEN ACCESS

Edited by:

Nicola Pavan,

University of Trieste, Italy

Reviewed by:

Fabrizio Di Maida,

Careggi University Hospital, Italy

Charles Doarn,

University of Cincinnati, United States

Anthony Bokolo,

Norwegian University of Science and

Technology, Norway

*Correspondence:

Albert El-Haj

ae67@aub.edu.Ib

tThese authors have contributed equally to this work and share first

authorship

Specialty section:

This article was submitted to

Genitourinary Surgery,

a section of the journal

Frontiers in Surgery

Received: 09 November 2021

Accepted: 31 January 2022

Published: 22 February 2022

Citation:

Ayoub CH, El-Asmar JM, Abdulfattah S and El-Hajj A (2022)

Telemedicine and Telementoring in Urology: A Glimpse of the Past and a

Leap Into the Future.

Front. Surg. 9:811749.

doi: 10.3389/fsurg.2022.811749

\section{Telemedicine and Telementoring in Urology: A Glimpse of the Past and a Leap Into the Future}

\author{
Christian Habib Ayoub ${ }^{1 \dagger}$, Jose M. El-Asmar ${ }^{1 \dagger}$, Suhaib Abdulfattah ${ }^{2}$ and Albert El-Hajj ${ }^{1 *}$ \\ ${ }^{1}$ Department of Surgery, Division of Urology, American University of Beirut Medical Center, Beirut, Lebanon, ${ }^{2}$ American \\ University of Beirut Medical School, American University of Beirut, Beirut, Lebanon
}

Telemedicine is the process of utilizing telecommunications and digital relay to perform, teach, or share medical knowledge. The digital era eased the incorporation of telemedicine to different areas of medical care, including the surgical care of Urologic patient mainly through telementoring, telesurgery, and telerobotics. Over the years, Telemedicine has played an integral part in a physicians' ability to provide high quality medical care to remote patients, as well as serve as an educational tool for trainee physicians, in the form of telementoring. During the COVID-19 pandemic, telemedicine has played a vital role in combatting the health implications of confinements. Challenges of telemedicine implementation include cost, ethical considerations, security, bandwidth, latency, legal, and licensure difficulties. Nevertheless, the future of telemedicine, specifically telementoring, promises several improvements and innovative advancements that aim to bridge the gap in technological divides of urologic care. In this review, we build on what is already known about telemedicine focusing specifically on aspects related to telementoring, telestration, and telesurgery. Furthermore, we discuss its historical role in healthcare with a special emphasis on current and future use in urology.

Keywords: telemedicine, telementoring, urology, robotic, telerobotic, telecommunications, COVID-19, education

\section{INTRODUCTION}

Telemedicine can be defined as any technology or technique that uses telecommunications or any form of remote interactions for medical interventions (1); it is the virtual conveyance of healthcare related information between two distinct sites. Telemedicine encompasses several subcategories that include but are not limited to: Telecommunications, Telementoring and Telesurgery (2). Telementoring is further divided into Teleproctoring, Telestration and Teleassistance (2). Teleproctoring entails verbal guidance by a mentor to a trainee. Telestration enhances the interaction by allowing the mentor to indicate or draw on a live feed during an intervention or procedure, while Teleassistance permits the remote surgeon to have direct access to some of the instruments involved in the procedure (2). On the other hand, telesurgery is the independent conduction of surgery by a remote surgeon $(3,4)$. In the past years, the application of telemedicine has seen significant growth among healthcare workers. In 2020, the European telemedicine market was valued at $\$ 10.6$ million, and it is expected to reach around $\$ 30$ million by $2026^{1}$ (5). Approximately 15 million Americans

${ }^{1}$ https://www.mordorintelligence.com/industry-reports/global-telemedicine-market-industry 
receive remote medical assistance yearly (6). In this review, we build on what is already known about telemedicine focusing specifically on aspects related to telementoring, telestration, and telesurgery. The purpose of this review is to highlight its historical role in healthcare with a special emphasis on current and future use in urology. We further discuss the role of telementoring and telesurgery in everyday urologic care emphasizing their benefits and limitations.

\section{METHODS}

We performed an extensive search in Google Scholar, PubMed and MedLine using the terms "urology", "urologic surgical procedures", "urolog* method*" combined with one of the following terms: "telemedicine", "telemedic*", "telecommunicat*”, "telestration", "telerobot*", "remote mentoring", and "education". For the section on COVID-19 we added to our previous search "COVID-19*, "COVID" and "Pandemic". We inlcluded original articles, systematic reviews, and brief reviews written in the English langauge. We focused on addressing articles that discussed telementoring and telesurgery. Articles about telerounding, teleconsultations, and televisits were beyond the scope of this paper, hence were excluded. A total of 102 articles were screened of which 94 were found to be relevant and 74 were included in this review whereas 9 of those addressed the topic in relation to COVID-19 (Figure 1). We sough to write a brief narrative, non-systematic review discussing telementoring, telestration, and telesurgery in urology.

\section{History of Telemedicine}

An early application of Telemedicine dates back to 1903 when Willem Einthoven, father of electrocardiography, successfully achieved a trans-telephonic transmission of an electrocardiograph to a distant hospital (7). Since 1929, laparoscopic techniques were introduced, and their use was first investigated in Urology and General surgery (8). The adoption of laparoscopic and robotic techniques in urologic surgeries eased the use of distant interactions between two remote sites. The invention of Automated Endoscopic System for Optimal Positioning (AESOP) in 1993 (9), Probot in 1996 (10, 11), Percutaneous Access to the Kidney (PACKY) in 1997 (12), Zeus, and later the DaVinci robots made such interactions possible (13). Those inventions paved the way for laying the foundations of telesurgical platforms.

One of the earliest uses of telementoring, specifically telestration, was done by Moore et al. (14) in 1995. An experienced surgeon mentored a trainee, 1,000 feet apart, to perform 23 laparoscopic procedures by utilizing high resolution video feeds, 2-way audio connections, telestration

Abbreviations: COVID-19, Coronavirus Disease of 2019; AESOP, Automated Endoscopic System for Optimal Positioning; PAKY-RCM, Percutaneous Access to the Kidney Remote Center of Motion; ISDN, Integrated Services Digital Network; AUA, American Urology Association; ACA, Affordable Act Care; HITECH, Health Information Technology for Economic and Clinical Health; HIP, Health Informatics Professional; ITRC, Identity Theft Resource Center. platform, and AESOP robot. 1 year later, a similar setup was developed for telesurgical consultations and was successfully used to perform 6 complex laparoscopic surgeries (15). In 1996, the first attempt at teleassistance was done by Schulam et al. (16) who utilized a newer version of the AESOP (1,000 TS) robot granting the remote surgeon full control of the electrocautery devices during the procedure. All these attempts were successful and showed comparable results between on site and distant telementoring (14-16). In the following years, further improvements to the field of telementoring and telemedicine were introduced. Between 19982000 , Bove et al. (1) successfully performed 17 transcontinental telementored and telestrated procedures between Italy and USA using AESOP and PAKY through Integrated Services Digital Network (ISDN) telephone lines rather than fiber optic lines. This aimed at simplifying connectivity issues between the sites. Nevertheless, in the initial pilot study, 5 out of 17 cases faced connection difficulties to the remote site, due to high bandwidth, halting the procedure. The authors concluded that a full adoption of telementoring procedures could only be established after significant advancements in the telecommunication systems (1).

The introduction of Zeus and DaVinci robots marked a breakthrough in the use of telesurgery. These robots received FDA approval in early 2,000 and were quickly used in general and urologic surgeries. The first robotic tele-cholecystectomy was successfully performed by Jacques Marescaux et al. (17) using the Zeus Robot and transatlantic optical fiber network between France and New York, known as "The Lindbergh Operation". It was deemed as a successful, reliable procedure with no perceived lag (17). In urology, Sterbis et al. (18) used public internet connectivity to perform the first transcontinental use of a DaVinci robot to perform 4 right nephrectomies on Porcine models that were 1,300 and 2,400 miles away, latency times was minimal between 450 to $900 \mathrm{~ms}$. These historical events paved the way for the integration of telemedicine in every day medical practice.

\section{Telementoring: Teleproctoring and Telestration}

The ability to safely guide a trainee during the learning phase of a procedure has been at the core of surgical education. To do so remotely is the essence of Telementoring. Telementoring encompasses Teleproctoring and Telestration.

Teleproctoring requires a trainer and a trainee to be connected through an interface that allows direct communication between the two. The interface requires two essential components: a digital platform and a connection between the two sites.

Connections either depend on cable or wireless systems as means of information relay. Over the years, those systems have developed to enhance the learning experience. At first, an ISDN and dedicated trunk communication lines were used $(1,16)$, followed by the use of cable networks in the form of dedicated fiber optic channels that provided a faster bandwidth and a decreased lag time (17). Further improvements in the field of 


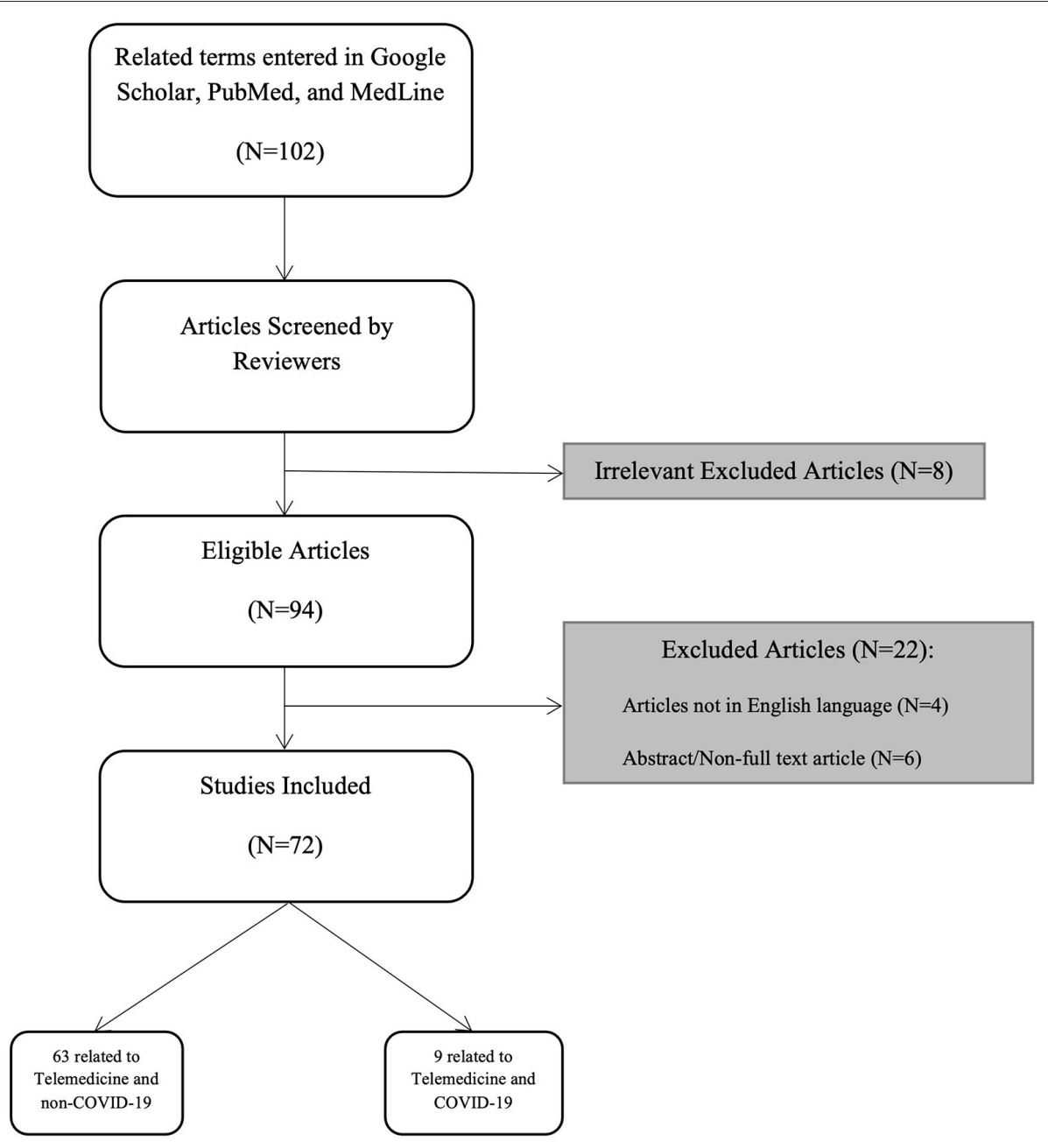

FIGURE 1 | Flowchart illustrating the search strategy used in this review.

telecommunications led to the use of wireless networks such as local internet connectivity. They were considered a means for practical and inexpensive telemedical communications (18). Today, wireless internet networks have rapidly evolved into providing unprecedent levels of data transfer speeds leading to the 5th generation technology standard for broadband of cellular networks, known as 5G. Mobile cellular networks satellites have been efficiently used in various forms of telemedicine with minimal connectivity lag (3).

Digital platforms of communication for teleproctoring and telestration have been developed to facilitate effective twoway communications between a mentor and his trainee. Such interfaces include a telementoring platform developed by Intuitive Surgical Inc. (Sunnyvale, CA, USA), called Connect ${ }^{\mathrm{TM}}$ and a telemetry system developed in Boston, London, and Beirut, called Proximie ${ }^{\mathrm{TM}}$. The aim is to permit virtual scrubbing of mentors to guide trainee surgeons by assisting in all aspects of the procedure including incision sites, anatomical identification etc.
Shin et al. (19) used Connect to mentor 55 robotic prostate and renal surgeries of which 29 were onsite and 26 were telementored. The interface provided effective telestration on the operative screen using the robotic console over a hospitalbased internet connectivity. The results of this study showed that telementoring was simple and effective allowing for minimal blood loss with similar operative times between telementored and non-telementored cases. Furthermore, higher satisfaction rates were reported by surgeons and trainees where mentors preferred remote over in-person interaction (19).

Similarly, a more recent study using another telementoring interface called Proximie compared perioperative outcomes of 59 cases of Aquablation, a novel robotic procedure to treat Benign Prostatic Obstruction, of which 39 were on site while 21 were telementored. This platform allows augmented reality live videos stream, low bandwidth internet connectivity, 2-way audio-video relay with zoom and encrypted web servers. It also allows the surgeon to draw on the displayed image to highlight important structures and anatomic landmarks. Similar operative outcomes 
and complications were seen between telementored cases and on-site cases demonstrating the practicality of such interfaces in teleproctored cases (20).

With innovative digital platforms and connections comes the ability to illustrate surgical steps on the surgical screen. Telestration adds a new dimension to telementoring whereby a trainer can telestrate his operative thoughts onto the field by identifying, delineating, or drawing on the displayed image to highlight important structures and anatomic landmarks during a procedure. This direct two-way interaction offered by Telestration has been shown to significantly improve the teleproctoring experience $(4,19)$.

\section{Telesurgery and Telerobotics}

Telesurgery is defined as the ability to independently perform a surgical procedure remotely (9). On the other hand, tele-robotics is a subtype of telesurgery whereby robotic instruments are used to perform the surgery. In 1995, the field of Urology witnessed the first application of a full telesurgical approach when Rovetta et al. (26) performed the first telesurgical transrectal ultrasoundguided biopsy of the prostate. Initially, laparoscopic equipment were utilized in telesurgical procedures using the AESOP robot. The remote surgeon was only limited to camera control and some laparoscopic or electrocautery functions $(1,16)$. With the introduction of the Da Vinci robot, the remote surgeon was able to manipulate more equipment and in some cases perform a full telerobotic surgery (18). Telerobotic surgeries in Urology were initially introduced using the Da Vinci surgical system to perform remote nephrectomies on distant porcine models (18). While in fact one of the first examples of telerobotic surgery took place in the year 2002 between Baltimore and Munich during a laparoscopic renal cyst ablation (22). In 2020, the Da Vinci surgical system was used to perform 1.25 million procedures, a $1 \%$ growth compared to 2019 (23). It is expected that the robotic market in urology will witness a growth of $11.7 \%$ by $2027^{2}$ (24). Today, further improvements in surgical robots allowed the introduction of competitive systems to the market such as Senhace, Revo-I, Versius, Avater, Hinotori, and most recently the Hugo RAS (25). Data on these systems are still not well-established and human trials are ongoing. Nevertheless, promising advances in the telerobotics sector are expected.

\section{DISCUSSION}

\section{Benefits to Physician and Patient}

Teleproctoring and Telestration have various advantages related to physician availability, omission of travel costs, combatting travel related restrictions, and emergency interventions specially in conflict areas with underdeveloped healthcare systems (Table 1). Rogers et al. (21) showed that telementoring between a community-based hospital and a trauma center resulted in 7\% lifesaving consultations and more than $80 \%$ approval rating for improved patient care. In regards to the immediate aid of areas of conflict, telementoring was the sole solution for performing

\footnotetext{
${ }^{2} \mathrm{https} / /$ www.databridgemarketresearch.com/reports/global-urology-roboticsurgery-market
}

TABLE 1 | Benefits of telementoring and telesurgery.
Increases physician availability

especially in emergencies

Decreases travel hurdles and costs

Facilitates travel related

Allows widespread dissemination of high-quality healthcare especially in areas with underdeveloped healthcare complications and restrictions
Improves resident training by allowing state-of-the-art surgical taught remotely

Decreases the impact of pandemics and worldwide health crisis on delivering healthcare

Decreases educational gaps by allowing conferences, lectures and rounds to occur remotely

Increases interactions between physician and trainee by allowing physician to clearly telestrate crucial surgical steps in the trainee's surgical view techniques and procedures to be

a complex hand reconstruction surgery for a blast injury patient in Gaza (4). It is estimated that five billion people do not have access to safe and affordable healthcare and that only $6 \%$ of the world's annual surgical procedures occur in the poorest countries (6). Using telementoring and telesurgery, patients in remote areas and underdeveloped countries can benefit from high quality healthcare. Physicians have shown various levels of satisfaction using telemedicine. In one study, mentors significantly favored remote telestration over in-room telestration (19). Furthermore, a survey study addressing the use of telementoring as a form of communication between residents and faculty physicians, revealed that most respondents expressed a moderate and strong agreement regarding the positive impact of telementoring in improving their interaction for the benefit of patients (27).

\section{Telemedicine in Coronavirus 2019 Pandemic}

In 2019, SARS-CoV-2, known as COVID-19, emerged in the city of Wuhan, China, spreading across all continents before being declared as a worldwide pandemic on 11 March 2020. This caused a major turning point by limiting human interactions affecting both their physical and mental well-being (28). COVID19 presented various challenges to the public, administrative and healthcare sector (29).

COVID-19 left a drastic impact on the healthcare sector affecting the worldwide health care economy, as well as the physical and mental well-being of healthcare professionals (29). In a scoping review of the impact of COVID-19 on education and training, junior medical staff reported that the pandemic significantly limited their educational activities and training quests (30). This was mainly due to the sharp reduction of nonCOVID-19 related patient encounters. (31). Urologist's interest in telemedicine increased during the pandemic from 43.7 to $80.8 \%$ with $81 \%$ interested to continue using telemedicine in their practice (32). Telemedicine was adopted in the pandemic as an easy and convenient alternative to mentor procedures without the need for travel. Furthermore, the American Urological Association urged urologists to optimize the use of telemedicine as it ensured the safety and well-being of their patients and 


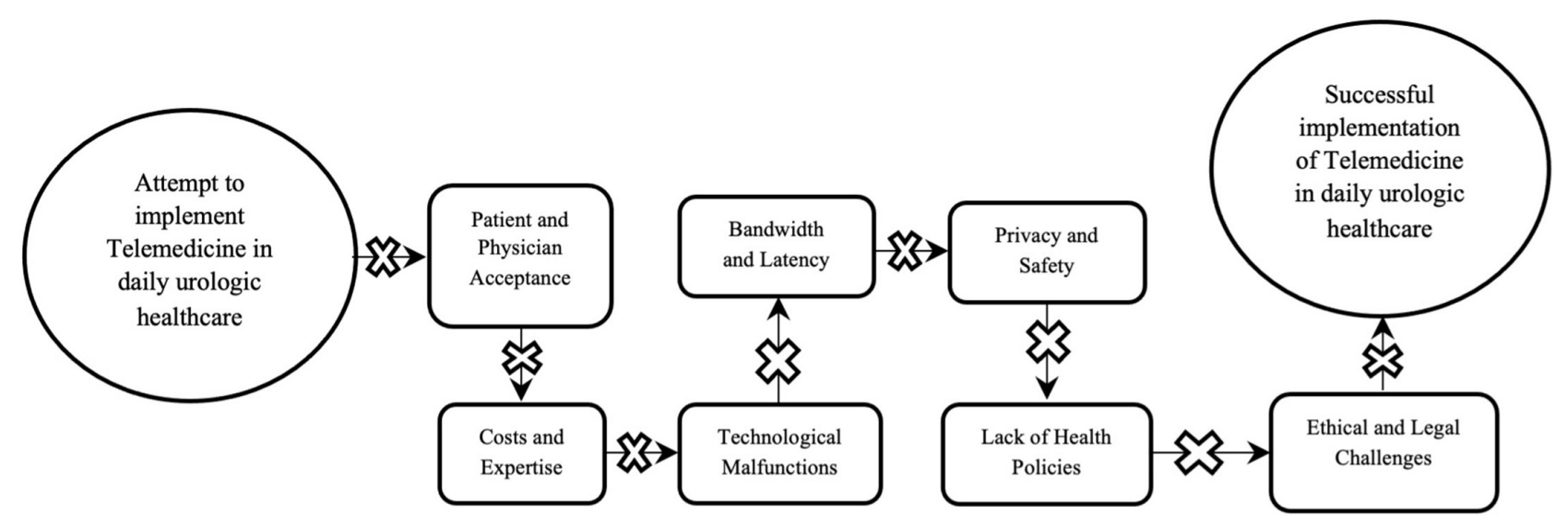

FIGURE 2 | Illustration depicting the obstacles of implementing telemedicine in urology.

workforce (33). An example of institutional use of telemedicine during the pandemic was the adoption of remote triaging by symptom screening (cough, sore throat, fever, cold etc.) and epidemiological history taking (contact with a positive case) over the phone prior to patient presentation to the hospital (34). In addition, there was a heavy reliance on virtual softwares such as Microsoft Teams, Zoom, Cisco, Webex and Skype that were extensively used to bridge the gap between physicians and patients. Bokolo et al. $(35,36)$ suggested a step-by-step workflow model that orchestrates remote consultation. This enabled patients to receive medical attention and schedule follow-up appointments. In fact, authors predicted their utility to last long after the end of the pandemic (35). In addition, efforts were made to lay the foundations for a remote interaction that is safe and governed by a set of practice guidelines and recommendations (36). Other studies demonstrated the successful use of telemedicine in selected patients during COVID-19 in the diagnosis, management, and follow-up care of oncologic and non-oncologic urologic diseases (37).

\section{Telemedicine and Education/Training}

In the wake of the COVID-19 pandemic, surgical residents reported a significant decline in their educational and training exposure $(30,38)$. Such decline was due to a decreased number of patient case load, as well as the cancellation of elective surgeries (39). In addition, educational activities such as lectures, grand rounds, and conferences were halted. Telemedicine aimed to combat those repercussions through offering an alternative means of telecommunication. Furthermore, physicians travel long distances to attend conferences, symposiums, or hands-on training courses to be up to date with the latest guidelines and novelties in the field. This leads to an increase in travel and logistical expenses. Telemedicine offers a way to ease knowledge gaps and make surgical training accessible without the need for travel (40).

As was previously stated, telemedicine can facilitate training collaborations between remote institutions allowing experienced surgeons to guide junior doctors in performing complex surgeries
(41). For instance, one study demonstrated that telementored residents performed as well as non-telementored residents during surgical training (42). Annual caseload affects surgical proficiency whereby an increase in case load increases proficiency (43). Telemedicine can fill the case load shortage through allowing urology trainees to virtually scrub in on cases to enhance their case load exposure. In addition, surgeons seeking mastery of minimally invasive surgeries can immensely benefit from telemedicine. For instance, the learning curve of a radical prostatectomy is around 250 cases, while more than $80 \%$ of surgeons perform less than 10 cases a year (44). As such, the ability to safely master this procedure can make use of a guiding hand that is able to remotely assist when needed. Telemedicine can act as the bridging gap for the occasional need of an expert opinion to surpass a challenging surgical step.

\section{Challenges of Telemedicine}

Telemedicine was found to be useful and effective in healthcare settings; nevertheless, it presents with limitations and challenges (Figure 2). Telemedicine is yet to be widely accepted in the urological community due to several limitations pertaining to patient and physician acceptance, licensure and liability, costs, safety, ethical considerations, and changes in workflow $(45,46)$.

In some cases, patients reported differences in quality of care and hesitancy in using such novel techniques in healthcare (45). On the other hand, healthcare providers reported lack of experience in using these technologies, resistance toward detachment from physical patient interactions, and inconvenient changes in staff and workflow (45). In addition, physicians faced licensure dilemmas and financial obstacles due to telemedical use across borders leading to an inadequate distribution of liability between mentor and trainee (47).

Telemedical practices incur heavy costs. As an example, robotic devices require an investment and running cost of around $800,000 \$$ and another $100,000 \$$ per year in maintenance fees. Not many hospitals can afford such expenses (48). On the other hand, on-site-mentoring incurs travel costs, patient lost time, and physical presence of the physician, whereas telementoring 
allows these expenses to be offset at the expense of buying tools for telecommunications (49). Further studies are required to determine the cost-benefit relationship of telemedicine.

Technology presents with various pitfalls. Machines are vulnerable to malfunction and in certain situations, such malfunctions could be catastrophic. Bove et al. performed 17 telementored procedures using AESOP and PAKY between Rome and the USA. In these 17 procedures, five were dropped from telementoring due to connection difficulties, two converted to open due to intra operative complications, and two faced intraoperative technical robotic malfunctions (1). Furthermore, various telementored and telesurgical attempts reported equipment malfunction and internet instability causing complications in their attempts $(18,50,51)$. Bandwidth and latency hurdles are also limitations of telesurgical and telementored procedures. The amount of delay, connection stability, and integrity of the surgical/telementored experience relies on an adequate bandwidth with minimal latency. To provide minimal latency times, bandwidth, known as the rate of data transfer between two points, should be sufficient. Previously, bandwidth speeds depended on the type of data connection, distance of data transfer and overall traffic in the circuit. Nowadays, due to $5 \mathrm{G}$, bandwidth speeds have reached $1 \mathrm{~Gb} / \mathrm{s}$ allowing short latency times and almost instant surgical and telementored relays (3). The exact amount of latency permitted is yet to be established, but various studies suggested that in order to maintain surgical performance latency times should not exceed a range of $330-450 \mathrm{~ms}(18,41,52)$. A final drawback to telemedicine is privacy and safety. Hacking and cyberattacks are concerns to tele-robotic networks, leading to concerns for the safety and vulnerability of data transfer (53). Breaches to these networks allow access to confidential patient data and privacy. Faster wireless connections, reliable firewalls, and virtual private networks (VPNs) are amongst the safety implementations needed to resolve these concerns.

\section{Telemedicine and Health Policy}

Novel approaches to healthcare, such as telemedical practices should be defined and regulated by regional and international policies. Health policies and telemedicine are interconnected and for telemedicine to be effective, regulated and fair between patients, health policies should be drafted and applied. Telemedicine offers innumerable healthcare advantages including easy access, convenience, self-efficacy, cost-cutting, and improvement in the overall population health (54). These advantages should encourage policy makers to enforce regulations that minimize the obstacles of telemedicine. Licensure between different states is one of the main obstacles faced (54). In the United States, the Federation of State Medical Licensing Board has erected the Interstate Medical Licensing Compacture that aimed to combat interstate variability by ensuring expedited licensure for the remote physician $(55,56)$. In addition, state specific policies were also drafted by the American Telemedicine Resource Center in an attempt to define and unify telemedical cross state interactions ${ }^{3}$ (57). Nevertheless, there

${ }^{3}$ https://www.americantelemed.org/ exists a lack of unified and robust national and international policies allowing providers to provide healthcare across borders (54). Other obstacles to be tackled by health policy makers are reimbursement controversies and patient information privacy (54). Policies such as the Affordable Act Care (ACA) and Health Information Technology for Economic and Clinical Health (HITECH) Act have attempted to address such limitations by ensuring fair reimbursements and securing that patient information remains safe and confidential $(58,59)$. It is without doubt that health policies regulating telemedicine improved dramatically over the past couple of years, yet further improvements are still due.

\section{Legal Challenges of Telementoring and Telesurgery}

Telemedical practices such as telementoring and telesurgery are more effective when allowed to supersede limitations imposed by geographical boundaries. The fact that telemedical encounters often occur across borders lead to several legal dilemmas. In the United States, state specific physician licensing is a major legal hurdle against the adoption of telemedicine across state borders; hence, the ability to treat patients or telementor procedures require different licenses in different states (45). As an illustration, several states mandate that remote physicians need to hold state specific licenses prior to treating patients in those states (60). Furthermore, some states restrict telemedical encounters to follow-up visits only, hence patients must first attend to an in-person visit before telemedical encounters are allowed (46). It is worth noting that health policies and licensing organizations have allowed expedited licensure for remote physicians $(55,56)$. In fact, state specific policies and regulations for physicians treating patients across state borders can be found in the American Telemedicine Association Resource Center ${ }^{3}$ (57). However, there still lacks a unified legal reference to define multistate licensing and to organize cross-border physician practices $(49,61)$. On the other hand, telementoring and telesurgery pose serious ethical considerations in regards to the proper attainment of a patient's informed consent. Consent for the telementored or teleoperated procedure should be clear, specific, and detailed informing the patient of the risks and benefits of such a procedure $(62,63)$. The procedure or intervention should be clearly described making sure that the explanation is clear, understandable, unambiguous and explicitly addresses the shortcomings of a tele approach $(63,64)$. Further legal obstacles that can complicate a telemedical procedure includes data protection, physician malpractice, and physician liability (61). Some authors believe that health informatics professionals (HIPs) who are responsible for the management, security, and implementation of healthcare informatics systems should be accountable by laws and codes in regards to data protection and breaches related to telemedical practices (65). A report by the Identity Theft Resource Center (ITRC) showed that the medical/healthcare sector contributed to $34.5 \%$ of the total amount of data breach incidents (66), thus data transfer over virtual networks during telementoring and telesurgery render the process significantly hackable. Several legislations and laws were 
drafted to protect patient data and security such as the General Data Protection Regulation, Directive 2011/24/EU, and others (67). Nevertheless, gaps regarding medical liability, security, and legal concerns need to be addressed and standardized prior to universal adoption of telemedicine (67).

\section{FUTURE DIRECTIONS}

The digitalization of todays' world enables a future global integration of telemedicine. Telemedicine and Telementoring have a prosperous future based on the initial studies that proved their efficiency and ease of implementation. Subsequently, telementoring using augmented reality (AR) and wearable equipment will be possible. Such attempts have already been materialized using holographic glasses such as Google Glass and Microsoft HoloLense. These models were found to be effective in remote telementoring yet require human trials to further assess their efficiency $(68,69)$. The telesurgical and telerobotic industry witnessed the introduction of various novel robotic systems to the market. These new robots feature significant improvements and advances in their setup. Some improvements include single port entries, smaller patient carts, open surgeon consoles with augmented reality, haptic feedback, and separated robotic arms (25). These new robots have been found to be effective in many surgeries and have provided benefits as compared to more traditional machines (70-72). Nevertheless, further trials and studies are required to prove the advantages of such changes to telerobotics.

\section{LIMITATIONS}

This review has several limitations. To start, we limited our search to telementoring, telesurgery, and telerobotics. Hence, we have left out other uses of telemedicine in urology such as teleconsultations, telehealth, and telerounding. The uses of telehealth were only mentioned when discussing the role of

\section{REFERENCES}

1. Bove P, Stoianovici D, Micali S, Patriciu A, Grassi N, Jarrett TW, et al. Is telesurgery a new reality? Our experience with laparoscopic and percutaneous procedures. J Endourol. (2003) 17:137-42. doi: 10.1089/0892779033216 18699

2. Challacombe B, Kavoussi L, Patriciu A, Stoianovici D, Dasgupta P. Technology insight: telementoring and telesurgery in urology. Nat Clin Pract Urol. (2006) 3:611-7. doi: $10.1038 /$ ncpuro0626

3. Zheng J, Wang Y, Zhang J, Guo W, Yang X, Luo L, et al. 5G ultra-remote robot-assisted laparoscopic surgery in China. Surg Endosc. (2020) 34:517280. doi: 10.1007/s00464-020-07823-x

4. Greenfield MJ, Luck J, Billingsley ML, Heyes R, Smith OJ, Mosahebi A, et al. Demonstration of the effectiveness of augmented reality telesurgery in complex hand reconstruction in Gaza. Plast Reconstr Surg Glob Open. (2018) 6:e1708. doi: 10.1097/GOX.0000000000001708

5. Intelligence M: Global telemedicine market - growth, trends and forecasts 2021-2026. Available online at: http://www.mordorintelligence.com/ industry-reports/global-telemedicine-market-industry.

6. Wachs JP, Kirkpatrick AW, Tisherman SA. Procedural telementoring in rural, underdeveloped, and austere settings: origins, present telemedicine during the COVID-19 pandemic. The aim was to focus on telementoring and telesurgery, two important pillars in remote medical care; hence, refraining from further exploring into the uses and implications of the other subtypes of telemedical care. The following manuscript is a brief narrative, non-systematic review of what is already known regarding telemedicine in urology. Broader categories of telemedicine and related publications were beyond the scope of this paper. In addition, we searched three major databases: PUBMED, MEDLINE and Google Scholar leaving out other databases that might have contributed to our paper.

\section{CONCLUSION}

Telemedicine has the potential to break geographical limitations of medical care. It is an exciting and promising field with boundless opportunities. The implementation of telemedical practices can greatly impact the quality of patient care, in addition it serves as a tool for enhancing the training of resident physicians and combatting pandemic related confinements. There are still several barriers impeding a full integration of telemedicine such as cost, ethical considerations, security, bandwidth, latency, and licensure difficulties. Nevertheless, the future of telemedicine, specifically telementoring and telesurgery, promise several improvements to tackle those barriers.

\section{AUTHOR CONTRIBUTIONS}

CA conceptualized the study, retrieved, read, and summarized the articles, and wrote the manuscript. JE-A conceptualized the study, retrieved and summarized the articles, and wrote the manuscript. SA retrieved and summarized the articles and wrote the manuscript. $\mathrm{AE}-\mathrm{H}$ reviewed and edited the manuscript and supervised the review and writing process. All authors contributed to the article and approved the submitted version. challenges, and future perspectives. Annu Rev Biomed Eng. (2021) 23:115-39. doi: 10.1146/annurev-bioeng-083120-023315

7. Barold SS. Willem Einthoven and the birth of clinical electrocardiography a 100 years ago. Card Electrophysiol Rev. (2003) 7:99-104. doi: 10.1023/a:1023667812925

8. Athanasiadis G, Bourdoumis A, Masood J. Is it the end for urologic pelvic laparoscopic surgery? Surg Laparosc Endosc Percutan Tech. (2017) 27:13946. doi: 10.1097/SLE.0000000000000406

9. Sim HG, Yip SK, Cheng CW. Equipment and technology in surgical robotics. World J Urol. (2006) 24:128-35. doi: 10.1007/s00345-006-0070-6

10. Davies BL, Hibberd RD, Ng WS, Timoney AG, Wickham JE. The development of a surgeon robot for prostatectomies. Proc Inst Mech Eng H. (1991) 205:358. doi: 10.1243/PIME_PROC_1991_205_259_02

11. Harris SJ, Arambula-Cosio F, Mei Q, Hibberd RD, Davies BL, Wickham JE, et al. The probot-an active robot for prostate resection. Proc Inst Mech Eng H. (1997) 211:317-25. doi: 10.1243/0954411971534449

12. Cadeddu JA, Bzostek A, Schreiner S, Barnes AC, Roberts WW, Anderson $\mathrm{JH}$, et al. A robotic system for percutaneous renal access. J Urol. (1997) 158:1589-93. doi: 10.1097/00005392-199710000-00081

13. Challacombe BJ, Khan MS, Murphy D, Dasgupta P. The history of robotics in urology. World J Urol. (2006) 24:120-7. doi: 10.1007/s00345-006-0067-1 
14. Moore RG, Adams JB, Partin AW, Docimo SG, Kavoussi LR. Telementoring of laparoscopic procedures: initial clinical experience. Surg Endosc. (1996) 10:107-10. doi: 10.1007/BF00188353

15. Cheriff AD, Schulam PG, Docimo SG, Moore RG, Kavoussi LR. Telesurgical consultation. J Urol. (1996) 156:13913. doi: 10.1097/00005392-199610000-00038

16. Schulam PG, Docimo SG, Saleh W, Breitenbach C, Moore RG, Kavoussi L. Telesurgical mentoring. Initial clinical experience. Surg Endosc. (1997) 11:1001-5. doi: 10.1007/s004649900511

17. Marescaux J, Leroy J, Rubino F, Smith M, Vix M, Simone $\mathrm{M}$, et al. Transcontinental robot-assisted remote telesurgery: feasibility and potential applications. Ann Surg. (2002) 235:48792. doi: 10.1097/00000658-200204000-00005

18. Sterbis JR, Hanly EJ, Herman BC, Marohn MR, Broderick TJ, Shih SP, et al. Transcontinental telesurgical nephrectomy using the da Vinci robot in a porcine model. Urology. (2008) 71:971-3. doi: 10.1016/j.urology.2007.11.027

19. Shin DH, Dalag L, Azhar RA, Santomauro M, Satkunasivam R, Metcalfe C, et al. A novel interface for the telementoring of robotic surgery. BJU Int. (2015) 116:302-8. doi: 10.1111/bju.12985

20. El-Asmar JM, Labban M, El-Hajj A. Integration of aquablation through telemetry: an alternative to onsite proctoring?. World J Urol. (2021) 39:34739. doi: 10.1007/s00345-021-03603-x

21. Rovetta A, Sala R. Execution of robot-assisted biopsies within the clinical context. J Image Guid Surg. (1995) 1:280-7: doi: 10.1002/(SICI)1522-712X(1995)1:5<280::AID-IGS4>3.0.CO;2-6

22. Frimberger D, Kavoussi L, Stoianovici D, Adam C, Zaak D, Corvin S, et al. [Telerobotic surgery between Baltimore and Munich]. Urologe A. (2002) 41:489-92. doi: 10.1007/s00120-002-0197-z

23. Intuitive Surgical I. Annual Report 2020, Sunnyvale (CA):[132 p.]. Available online at: https://isrg.intuitive.com/static-files/80b10bf5-c1da-4ad3-bb0e$8 \mathrm{c} 595 \mathrm{e} 2 \mathrm{c} 712 \mathrm{c}$.

24. Global Urology Robotic Surgery Market - Industry Trends and Forecast to 2027: Data Bridge Market Research; 2020. Available online at: https://www. databridgemarketresearch.com/reports/global-urology-robotic-surgerymarket.

25. Koukourikis $\mathrm{P}$, Rha KH. Robotic surgical systems in urology: what is currently available?. Investig Clin Urol. (2021) 62:14-22. doi: 10.4111/icu.20200387

26. Rogers FB, Ricci M, Caputo M, Shackford S, Sartorelli K, Callas $\mathrm{P}$, et al. The use of telemedicine for real-time video consultation between trauma center and community hospital in a rural setting improves early trauma care: preliminary results. J Trauma. (2001) 51:103741. doi: 10.1097/00005373-200112000-00002

27. Mirone V, Creta M, Capece M, Celentano G, Califano G, Collà Ruvolo $\mathrm{C}$, et al. Telementoring for communication between residents and faculty physicians: results from a survey on attitudes and perceptions in an academic tertiary urology referral department in Italy. Archivio Italiano di Urologia e Andrologia. (2021) 93:450-4. doi: 10.4081/aiua.2021.4.450

28. Chriscaden K. Impact of COVID-19 on people's livelihoods, their health and our food systems. Joint statement by ILO, FAO, IFAD and WHO [Internet]. 2020. Available online at: https://www.who.int/news/item/13-10-2020impact-of-covid-19-on-people's-livelihoods-their-health-and-our-foodsystems.

29. Lakhani A, Sharma E, Gupta K, Kapila S, Gupta S. Corona virus (COVID-19) and its impact on health care workers. J Assoc Physicians India. (2020) 68:66-9.

30. Seifman MA, Fuzzard SK, To H, Nestel D. COVID-19 impact on junior doctor education and training: a scoping review. Postgrad Med J. (2021). doi: 10.1136/postgradmedj-2020-139575

31. Moynihan R, Sanders S, Michaleff ZA, Scott AM, Clark J, To EJ, et al. Impact of COVID-19 pandemic on utilisation of healthcare services: a systematic review. BMJ Open. (2021) 11:e045343. doi: 10.1136/bmjopen-2020-045343

32. Dubin JM, Wyant WA, Balaji NC, Ong WL, Kettache RH, Haffaf M, et al. Telemedicine usage among urologists during the COVID-19 pandemic: crosssectional study. J Med Internet Res. (2020) 22:e21875. doi: 10.2196/21875

33. Gadzinski AJ, Gore JL, Ellimoottil C, Odisho AY, Watts KL. Implementing Telemedicine in response to the COVID-19 pandemic. J Urol. (2020) 204:146. doi: 10.1097/JU.0000000000001033

34. Esperto F, Prata F, Civitella A, Pang KH, Marchioni M, Tuzzolo P, et al. Implementation and strategies to ensure adequate coordination within a urology department during the COVID-19 pandemic. Int Braz J Urol. (2020) 46(suppl.1):170-80. doi: 10.1590/s1677-5538.ibju.2020.s122

35. Bokolo AJ. Exploring the adoption of telemedicine and virtual software for care of outpatients during and after COVID-19 pandemic. Ir J Med Sci. (2021) 190:1-10. doi: 10.1007/s11845-020-02299-Z

36. Bokolo Anthony J. Use of telemedicine and virtual care for remote treatment in response to COVID-19 pandemic. J Med Syst. (2020) 44:132. doi: 10.1007/s10916-020-01596-5

37. Novara G, Checcucci E, Crestani A, Abrate A, Esperto F, Pavan N, et al Telehealth in urology: a systematic review of the literature. How Much Can Telemedicine Be Useful During and After the COVID-19 Pandemic? Eur Urol. (2020) 78:786-811. doi: 10.1016/j.eururo.2020.06.025

38. Amparore D, Claps F, Cacciamani GE, Esperto F, Fiori C, Liguori G, et al. Impact of the COVID-19 pandemic on urology residency training in Italy. Minerva Urol Nefrol. (2020) 72:505-9. doi: 10.23736/S0393-2249.20.03868-0

39. Tyagi V, Sharma AK, Bhandari M. Urological implications of SARS CoV-19. Can J Urol. (2020) 27:10205-12.

40. Snoswell CL, Taylor ML, Comans TA, Smith AC, Gray LC, Caffery LJ. Determining if telehealth can reduce health system costs: scoping review. J Med Internet Res. (2020) 22:e17298. doi: 10.2196/17298

41. Bruschi M, Micali S, Porpiglia F, Celia A, De Stefani S, Grande $\mathrm{M}$, et al. Laparoscopic telementored adrenalectomy: the Italian experience. Surg Endosc. (2005) 19:836-40. doi: 10.1007/s00464-0049124-2

42. Panait L, Rafiq A, Tomulescu V, Boanca C, Popescu I, Carbonell A, et al. Telementoring vs. on-site mentoring in virtual reality-based surgical training. Surg Endosc. (2006) 20:113-8. doi: 10.1007/s00464-005-0113-x

43. Santomauro M, Reina GA, Stroup SP. L'Esperance JO. Telementoring in robotic surgery. Curr Opin Urol. (2013) 23:141-5. doi: 10.1097/MOU.0b013e32835d4cc2

44. Savage CJ, Vickers AJ. Low annual caseloads of United States surgeons conducting radical prostatectomy. J Urol. (2009) 182:2677-9. doi: 10.1016/j.juro.2009.08.034

45. Castaneda P, Ellimoottil C. Current use of telehealth in urology: a review. World J Urol. (2020) 38:2377-84. doi: 10.1007/s00345-019-02882-9

46. Ellimoottil C, Skolarus T, Gettman M, Boxer R, Kutikov A, Lee BR, et al. Telemedicine in urology: state of the art. Urol. (2016) 94:106. doi: 10.1016/j.urology.2016.02.061

47. El-Sabawi B, Magee W III. The evolution of surgical telementoring: current applications and future directions. Ann Transl Med. (2016) 4:391. doi: 10.21037/atm.2016.10.04

48. Rassweiler J, Frede T. Robotics, telesurgery and telementoring-their position in modern urological laparoscopy. Arch Esp Urol. (2002) 55:610-28.

49. Hung AJ, Chen J, Shah A, Gill IS. Telementoring and telesurgery for minimally invasive procedures. J Urol. (2018) 199:35569. doi: 10.1016/j.juro.2017.06.082

50. Micali S, Virgili G, Vannozzi E, Grassi N, Jarrett TW, Bauer JJ, et al. Feasibility of telementoring between Baltimore (USA) and Rome (Italy): the first five cases. J Endourol. (2000) 14:493-6. doi: 10.1089/end.2000.14.493

51. Nguan CY, Morady R, Wang C, Harrison D, Browning D, Rayman R, et al. Robotic pyeloplasty using internet protocol and satellite network-based telesurgery. Int J Med Robot. (2008) 4:10-4. doi: 10.1002/rcs.173

52. Marescaux J, Leroy J, Gagner M, Rubino F, Mutter D, Vix M, et al. Transatlantic robot-assisted telesurgery. Nature. (2001) 413:379-80. doi: 10.1038/35096636

53. Bonaci T, Herron J, Yusuf T, Yan J, Kohno T, Chizeck H. To make a robo secure: an experimental analysis of cyber security threats against teleoperated surgical robots. arXiv Preprint arXiv:150404339. 2015.

54. Kruse CS, Williams K, Bohls J, Shamsi W. Telemedicine and health policy: asystematic review. Health Policy Technol. (2021) 10:209-29. doi: 10.1016/j.hlpt.2020.10.006

55. Kempen PM. The interstate medical licensure compact of the federation of state medical boards. J Am Physicians Surg. (2017) 22:2.

56. Silva E. III. The Interstate Medical Licensure Compact Journal of the American College of Radiology. (2015) 12:511. doi: 10.1016/j.jacr.2015.01.021

57. American Telemedicine Association. ATA Policy Principles. (2020). Available online at: https://www.americantelemed.org/policy/ (accessed January 16, 2022) 
58. Office of Civil Rights. HITECH Act Enforcement Interim Final Rule Updated 2017. Available online at: https://www.hhs.gov/hipaa/for-professionals/ special-topics/hitech-act-enforcement-interim-final-rule/index.html. (Accessed on January, 16, 2022).

59. Assistant Secretary for Public Affairs (ASPA). About the Affordable Care Act Updated (2021). Available online at: https://www.hhs.gov/healthcare/aboutthe-aca/index.html. (accessed on January, 15, 2022).

60. Hoffmann D, Rowthorn V. Legal impediments to the diffusion of telemedicine. J Health Care Law Policy. (2011) 14:54.

61. Nittari G, Khuman R, Baldoni S, Pallotta G, Battineni G, Sirignano A, et al. Telemedicine practice: review of the current ethical and legal challenges. Telemed J E Health. (2020) 26:1427-37. doi: 10.1089/tmj.2019.0158

62. Kramer GM, Kinn JT, Mishkind MC. Legal, regulatory, and risk management issues in the use of technology to deliver mental health care. Cogn Behav Pract. (2015) 22:258-68. doi: 10.1016/j.cbpra.2014.04.008

63. Ricci G, Pirillo I, Amenta F. Ethical challenges to medical assistance at sea. Marine Policy. (2017) 81:247-9. doi: 10.1016/j.marpol.2017.04.007

64. Botrugno C. Telemedicine in daily practice: addressing legal challenges while waiting for an EU regulatory framework. Health Policy Technol. (2018) 7:1316. doi: 10.1016/j.hlpt.2018.04.003

65. Kluge E-H, Lacroix P, Ruotsalainen P. Ethics certification of health information professionals. Yearb Med Inform. (2018) 27:37-40. doi: 10.1055/s-0038-1641196

66. Identity Theft Resource Center ITRC 2016 Data Breach Report: Identity Theft Resource Center; (2016). Available online at: https://www.idtheftcenter.org/ post/data-breaches-increase-40-percent-in-2016-finds-new-report-fromidentity-theft-resource-center-and-cyberscout/. (accessed on 23, January, 2022).

67. Raposo VL. Telemedicine: the legal framework (or the lack of it) in Europe. GMS Health Technol Assess. (2016)12: Doc03. doi: 10.3205/hta000126

68. Liu P, Li C, Xiao C, Zhang Z, Ma J, Gao J, et al. A wearable augmented reality navigation system for surgical telementoring based on microsoft hololens. Ann Biomed Eng. (2021) 49:287-98. doi: 10.1007/s10439-020-02538-5
69. Datta N, MacQueen IT, Schroeder AD, Wilson JJ, Espinoza JC, Wagner JP, et al. Wearable technology for global surgical teleproctoring. J Surg Educ. (2015) 72:1290-5. doi: 10.1016/j.jsurg.2015.07.004

70. Agarwal DK, Sharma V, Toussi A, Viers BR, Tollefson MK, Gettman MT, et al. Initial experience with da vinci single-port robot-assisted radical prostatectomies. Eur Urol. (2020) 77:373-9. doi: 10.1016/j.eururo.2019.04.001

71. Samalavicius NE, Janusonis V, Siaulys R, Jasenas M, Deduchovas O,

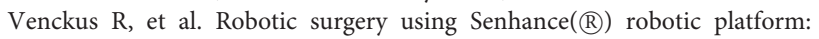
single center experience with first 100 cases. J Robot Surg. (2020) 14:3716. doi: 10.1007/s11701-019-01000-6

72. Chang KD, Abdel Raheem A, Choi YD, Chung BH, Rha KH. Retzius-sparing robot-assisted radical prostatectomy using the Revo-i robotic surgical system: surgical technique and results of the first human trial. BJU Int. (2018) 122:4418. doi: $10.1111 /$ bju. 14245

Conflict of Interest: The authors declare that the research was conducted in the absence of any commercial or financial relationships that could be construed as a potential conflict of interest.

Publisher's Note: All claims expressed in this article are solely those of the authors and do not necessarily represent those of their affiliated organizations, or those of the publisher, the editors and the reviewers. Any product that may be evaluated in this article, or claim that may be made by its manufacturer, is not guaranteed or endorsed by the publisher.

Copyright (C) 2022 Ayoub, El-Asmar, Abdulfattah and El-Hajj. This is an open-access article distributed under the terms of the Creative Commons Attribution License (CC $B Y)$. The use, distribution or reproduction in other forums is permitted, provided the original author(s) and the copyright owner(s) are credited and that the original publication in this journal is cited, in accordance with accepted academic practice. No use, distribution or reproduction is permitted which does not comply with these terms. 